\title{
Recognition and Approach Responses Toward Threatening Objects
}

\author{
Oliver Genschow, ${ }^{1}$ Arnd Florack, ${ }^{2}$ and Michaela Wänke ${ }^{1}$ \\ ${ }^{1}$ University of Mannheim, Germany, \\ ${ }^{2}$ Applied Social Psychology and Consumer Research, University of Vienna, Austria
}

\begin{abstract}
Previous research suggests that positive stimuli are often approached as well as recognized faster than negative stimuli. We argue that this effect does not hold if negative stimuli are associated with threat. Based on fear module theory (Öhman \& Mineka, 2001, 2003), we argue that individuals recognize threatening stimuli faster than positive stimuli because of a constant monitoring of the environment for threatening objects. Moreover, based on the assumption of a motivational account underlying approach-avoidance responses (Krieglmeyer \& Deutsch, 2010), we assume the recognition then directly evokes a careful and slow approach of threatening objects. Applying a response time task that measures approach movement and recognition times within the same task, we found that individuals recognize threatening pictures faster than positive pictures, but approach the threatening pictures slower than the positive pictures.
\end{abstract}

Keywords: fear module theory, threat, approach, recognition

Recent research has demonstrated that individuals recognize (Bargh, Chaiken, Govender, \& Pratto, 1992; Unkelbach, Fiedler, Bayer, Stegmüller, \& Danner, 2008; Unkelbach et al., 2010) and approach (Brendl, Markman, \& Messner, 2005; Chen \& Bargh, 1999; De Houwer, Crombez, Baeyens, \& Hermans, 2001; Krieglmeyer \& Deutsch, 2010; van Dantzig, Pecher, \& Zwaan, 2008) negative stimuli slower than positive stimuli. In this article, we hypothesize that this might not hold true when negativity is related to threat. Based on fear module theory (Öhman \& Mineka, 2001, 2003) and the assumption of a motivational account underlying approach-avoidance responses (Krieglmeyer \& Deutsch, 2010), we assume that individuals recognize threatening stimuli faster and approach them more slowly than positive stimuli.

\section{Responses Toward Positive and Negative Stimuli}

Research has repeatedly shown that individuals are faster at consciously recognizing positive stimuli than negative stimuli (Bargh et al., 1992; Unkelbach et al., 2008, 2010). For example, Bargh et al. (1992) found that positive attitude objects were evaluated more quickly than negative ones. Similarly, Unkelbach et al. (2010) found that individuals are faster at identifying positive compared to negative words. In what they referred to as the density hypothesis, the authors account for this phenomenon by arguing that positive information is more densely organized than negative information and that this increased density in turn leads to faster identification of positive stimuli.

Other research suggests that individuals approach positive stimuli faster than negative stimuli (Brendl et al., 2005; Chen \& Bargh, 1999; De Houwer et al., 2001; Krieglmeyer $\&$ Deutsch, 2010; van Dantzig et al., 2008). For example, Chen and Bargh (1999) demonstrated that participants responded faster toward positive stimuli by pulling (approach) than by pushing (avoidance) a lever, and faster toward negative stimuli by pushing (avoidance) a lever than by pulling (approach) it. Further research showed that the different response to positive and negative stimuli is not limited to a specific direction of movement (pulling or pushing), but rather depends on whether the direction of movement is framed as approach or avoidance (Eder \& Rothermund, 2008; Seibt, Neumann, Nussinson, \& Strack, 2008; see also, Brendl et al., 2005). In this sense, any movement can represent approach if the response elicits a virtual movement of the stimuli toward the participants, or avoidance if the response causes a virtual movement of the stimuli away from the participants (Morange \& Bloch, 1996; Seibt et al., 2008; van Dantzig et al., 2008; von Hofsten \& Rönnqvist, 1988). Irrespective of how approach and avoidance movements are defined, it is generally agreed that the recognition and evaluation of a stimulus activates a related behavioral schema (Eder \& Rothermund, 2008; Krieglmeyer \& Deutsch, 2010; Lavender \& Hommel, 2007; Strack \& Deutsch, 2004). That is, the recognition of positive stimuli activates behavioral approach schemata and the recognition of negative stimuli activates behavioral avoidance schemata. As a result, movements compatible to 
the activated schema can be performed faster than movements incompatible to the activated schema.

Notwithstanding the close link between recognition and activation of approach-avoidance movements, it is important to keep in mind that both represent different concepts and should be measured independently from each other. This is, unfortunately, rarely the case. The majority of approach-avoidance measures does not measure approach or avoidance movements separately from recognition, but rather lumps these components together. In the task applied by Chen and Bargh (1999), for example, the time between the presentation of a stimulus and the initial response with a lever was measured, but not the time for the movement of the lever from a point A to a point B. However, tasks that would differentiate between approach and recognition responses could provide important insights into how and when the two concepts are related, when they are independent, or when they are negatively correlated. Interestingly, only few methods, such as the task from Bamford and Ward (2008) or the Recognition and Behavioral Approach Task (RaBAT; Genschow et al., 2013), allow disentangling the speed of a movement from the time individuals need to recognize a stimulus. As we relied on the RaBAT in the present study, we now describe this task in more detail.

\section{The Recognition and Behavioral Approach Task (RaBAT)}

Participants working on the RaBAT (Genschow et al., 2013) have to press the space bar on a computer keyboard as soon as they have recognized a picture on the screen. Afterwards, they have to press a key closer to the screen (the Z key on a Swiss keyboard; Y key on English language keyboards) as fast as possible, which elicits a virtual movement of the picture toward the participants to give the illusion that the stimulus actually moves toward participants (cf. Seibt et al., 2008). Although many subprocesses (e.g., recollection, familiarity) may be involved in the recognition of objects (for research measuring event-related potentials, see Rugg \& Curran, 2007), recognition in the RaBAT is understood as the time between the appearance of a stimulus and the pressing of the space bar. The time between pressing the space bar and pressing the $\mathrm{Z}$ key is defined as the approach movement time. While recognition time is assumed to represent a neutral response resulting from a cognitive evaluation of the stimulus, approach movement time reflects a more meaningful component, because it causes the stimulus to come closer - visually at least. Similar to assumptions of affective primacy (e.g., Zajonc, 1984) and embodied cognition (e.g., Niedenthal, Barsalou, Winkielman, Krauth-Gruber, \& Ric, 2005), according to which embodied movements often precede conscious processes, the RaBAT assumes that the mere perception of a stimulus automatically activates a corresponding response. In line with the assumption of a motivational account underlying approach movements (Krieglmeyer \& Deutsch, 2010), we assume that the recognition of a stimulus facilitates such responses that are associated with fundamental needs of survival and nurturance. That is, positive stimuli facilitate and negative stimuli inhibit the execution of the approach movement. Beside the ability to measure recognition and approach separately another advantage of the RaBAT is its simplicity because individuals respond toward every stimulus in the same manner. Therefore, the RaBAT is not affected by task switching abilities, the interpretation of categories, or strategic answering behavior, which has been identified to influence the results of double categorization tasks like the implicit association test (e.g., Fiedler, Messner, \& Bluemke, 2006; Rothermund \& Wentura, 2004).

In line with previous research on recognition (Bargh et al., 1992; Unkelbach et al., 2008, 2010) and approach responses (Brendl et al., 2005; Chen \& Bargh, 1999; De Houwer et al., 2001; Krieglmeyer \& Deutsch, 2010; van Dantzig et al., 2008), a pilot study (Genschow et al., 2013) that applied the RaBAT found that participants recognized $(M=459 \mathrm{~ms})$ as well as approached $(M=$ $247 \mathrm{~ms})$ positive stimuli faster than negative stimuli $(M=$ $482 \mathrm{~ms} ; M=261.03 \mathrm{~ms}$, for recognition and approach, respectively). Extending this research, we suggest that (a) response latencies for recognition and approach movements may not always be positively correlated and (b) recognition may not always be slower for negative than for positive stimuli. Concretely, we hypothesized that negative stimuli that are related to threat should speed up - not slow down - recognition compared to positive stimuli and should slow down approach movements. This hypothesis highlights the importance of disentangling recognition from movement speed.

\section{Responses Toward Threatening Stimuli}

In their fear module theory, Öhman and Mineka (2001, 2003) propose a specific fear module that constantly monitors the environment for threat in an automatic and unconscious fashion. The authors assume that the fear module selectively processes those types of fear-relevant stimuli that have a long phylogenetic history of being associated with danger toward humans and their evolutionary ancestors and thus ensures a rapid detection of threatening stimuli. Furthermore, fear module theory supposes that the detection of fear-related objects evokes an in-born defense mechanism shaped by an evolutionary development, which ensures a functional preparation to take action and to deal with the situation properly (Mineka \& Öhman, 2002; Öhman, Flykt, \& Esteves, 2001; Williams, Watts, MacLeod, \& Mathews, 1988). An explanation of how such a functional behavioral response may work is offered by a motivational account underlying approach-avoidance impulses (Krieglmeyer \& Deutsch, 2010). According to this account, the evaluation of a stimulus induces a motivational orientation that prepares an organism to increase or decrease the distance between the self and the object in order to fulfill fundamental needs of survival and 
nurturance. Regarding threatening stimuli, it is plausible to assume that the evaluation elicits a fast avoidance or a slow approach response.

Individual findings on the detection of threatening stimuli are in line with the predictions of fear module theory. For instance, Öhman et al. (2001) demonstrated that in a visual search paradigm fear-relevant stimuli such as snakes or spiders are detected faster than fear-irrelevant stimuli such as flowers or mushrooms. This basic finding has been replicated among many other studies (e.g., Lipp \& Waters, 2007; Tipples, Young, Quinlan, Broks, \& Ellis, 2002). Moreover, research indicates that this pattern is already present at preschool age (LoBue \& DeLoache, 2008, 2010) and holds for modern threats such as syringes, guns, and knives (Blanchette, 2006; Brosch \& Sharma, 2005). Likewise there is also evidence from studies with approach-avoidance measures that indicate slowed down approach responses for threatening compared to positive stimuli (Krieglmeyer \& Deutsch, 2010; Rinck \& Becker, 2007). For example, in a study by Krieglmeyer and Deutsch (2010), participants were faster in approaching butterfly pictures than spider pictures and faster in avoiding spider than butterfly pictures. A more elegant test of the theoretical assumption that threatening stimuli lead to fast recognition and slow approach responses would involve the assessment of recognition and approach latencies within the same response sequence. As described before, the RaBAT (Genschow et al., 2013) allows doing this in a most convenient manner.

Because positive stimuli are generally recognized faster than negative stimuli (e.g., Genschow et al., 2013; Unkelbach et al., 2008), the hardest test of whether threat leads to a fast recognition is the comparison of threatening stimuli with positive stimuli (and not with neutral or negative stimuli). Therefore, the present study compares response latencies toward threatening and positive stimuli. As spiders are among the most common threatening targets that go along with warning states, such as fears and phobias (Fredrikson, Annas, Fischer, \& Wik, 1996) or disgust (Wiens, Peira, Golkar, \& Öhman, 2008), we used pictures of spiders as threatening stimuli and pictures of butterflies as positive stimuli.

The RaBAT is a task to measure approach, but not avoidance responses. Although it could, in principle, be adapted to measure avoidance latencies, we deliberately applied the task in its usual form, because avoidance responses would not provide us with insights into the research question of this study in which we were interested to show that threatening stimuli could speed up recognition and slow down approach movement speed. A finding that threatening stimuli speed up recognition as well as avoidance would not serve the goal to show that recognition and movement have to be differentiated.

\section{Method}

\section{Participants}

During an open day at the University of Basel, 74 visitors (26 male, 41 female, 7 not reported) with a mean age of $34.51(S D=18.13)$ volunteered to participate in a computer experiment. Eighteen participants were discarded from the analyses because they did not follow the instructions (16 persons used two fingers instead of one when working on the RaBAT), they did not understand the instructions (one person indicated that she did not understand the instructions at all, because she did not speak German), or their attention was not directed toward the task (one person was clearly distracted and interrupted by other visitors).

\section{Procedure}

Participants were tested in pairs or alone. After signing a statement of agreement, participants completed the RaBAT (Genschow et al., 2013), indicated basic demographic characteristics, and were then debriefed, thanked, and dismissed.

\section{Materials and Apparatus}

Thirty pictures (each $685 \times 549$ pixels) served as stimuli material. In line with Krieglmeyer and Deutsch (2010), the target pictures contained 10 pictures of spiders and 10 pictures of butterflies. ${ }^{1}$ As distractor items, we selected 10 pictures of stars. The size of the animals and the background of the pictures were similar between spider and butterfly pictures. The experiment was conducted on IBM-compatible (3,000+ processor) desktop computers with Windows XP as the system software. Participants viewed the screen from a distance of approximately $55 \mathrm{~cm}$ and responded on a Swiss keyboard.

1 To ensure that the spiders are generally perceived as more threatening than the butterflies, we conducted a pretest with 34 participants (24 male, 10 female) with a mean age of $25.71(S D=8.04)$. On a paper and pencil questionnaire, participants evaluated every animal on six 7-point bipolar scales. The negative endpoints of the scales were labeled with "frightening," "worrying," "scary," "threatening," "menacing," and "dangerous." The positive endpoints of the scale were labeled with "benign," "comforting," "confidential," "nonhazardous," "harmless," and "safe." We averaged the evaluations of the spider pictures (Cronbach's $\alpha=.98$ ) and the evaluations of the butterfly pictures (Cronbach's $\alpha=.99$ ) into a separate single scale. High values indicate high threat. To test whether participants evaluated spider pictures as more threatening than butterfly pictures, we conducted a $t$-test for dependent samples. The results indicate that participants evaluated spider pictures $(M=4.27, S D=1.46)$ as more threatening than butterfly pictures $(M=1.08, S D=1.31)$, $t(33)=-9.80, p<.001, d=2.30$. 


\section{Behavioral Recognition and Approach Task (RaBAT)}

We applied a RaBAT version that was already used by Genschow et al. (2013, Study 2). Participants were instructed to put, but not to press, one of their index fingers onto the space bar of the computer keyboard. After a fixation cross on the white screen was presented for a randomly selected time (500-2,000 ms), a picture on the screen appeared. Participants were told that as soon as they recognize the picture as either a butterfly or a spider, they have to press their finger onto the space bar and then to press the $\mathrm{Z}$ key on the Swiss keyboard (Y key on English language keyboards) as fast as possible. After pressing the $\mathrm{Z}$ key, the picture was zoomed in for 1,000 ms until it was $25 \%$ larger to illustrate the approach character of the movement (cf. Seibt et al., 2008; van Dantzig et al., 2008). After the zooming, participants were told to put their finger again on the space bar. Then, the next trial began. Similar to a go/no-go task (Nosek \& Banaji, 2001), we randomly included trials with distractor pictures to which participants were not allowed to react to ensure that participants only reacted when they really recognized a picture as a butterfly or as a spider.

The RaBAT contained a practice ( 15 trials) and a main phase (20 trials). In the practice phase, participants had to respond toward rectangles and triangles, but were not allowed to respond toward circles. In the main phase, participants responded toward butterflies and spiders, but were not allowed to respond toward stars. To prepare the data for the analysis, we discarded all latencies below $200 \mathrm{~ms}$ $(0.75 \%)$ and above $2,000 \mathrm{~ms}(0.55 \%)$ and then log-transformed all latencies. Afterwards, we computed mean recognition times (time between the appearance of a stimulus on the screen and the pressing of the space bar) and mean approach movement times (time between pressing the space bar and pressing the $\mathrm{Z}$ key) for butterflies as well as spiders. Although we used log-transformed latencies in our analyses, we report the untransformed latencies to ease the interpretation. ${ }^{2}$

\section{Results}

To test our hypotheses, we computed an ANOVA with two repeated measurement factors (response: recognition vs. approach movement; stimulus category: butterfly vs. spider) on the response times. As expected, the analysis yielded a significant interaction between response and stimulus category, $F(1,55)=6.88, p=.01, \eta_{\mathrm{p}}{ }^{2}=.11$ (see Figure 1 for means). Contrast analyses indicate that participants recognized spider pictures faster than butterfly pictures, $F(1,55)=4.73, \quad p=.03, \eta_{\mathrm{p}}^{2}=.08$. Conversely, participants approached butterfly pictures faster than spider pictures, $F(1,55)=4.00, p=.05, \eta_{\mathrm{p}}^{2}=.07 .^{3}$ In addition to the significant interaction effect, but less relevant for the

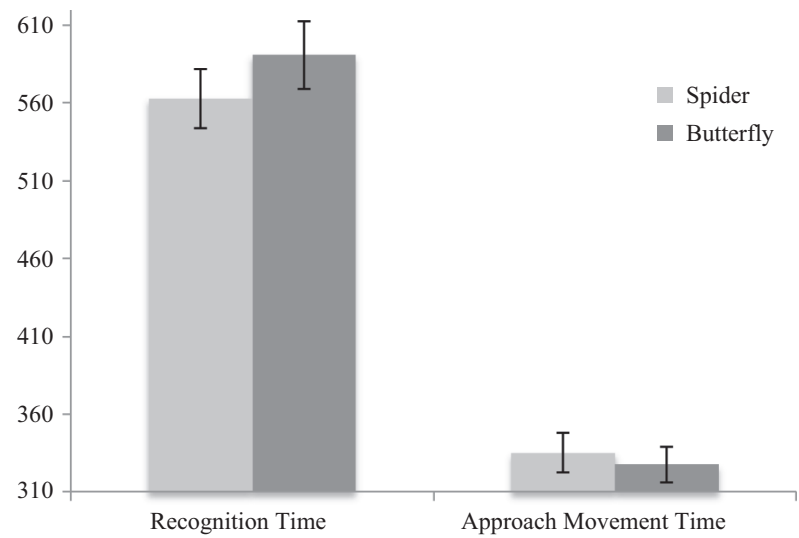

Figure 1. Mean recognition times and mean approach movement times (in $\mathrm{ms}$ ) toward spider and butterfly pictures (error bars indicate standard errors).

hypotheses, the analysis revealed a nonsignificant main effect for stimulus category, $F(1,55)=1.53, p=.22$, and a significant main effect for response, $F(1,55)=285.90$, $p<.001, \eta_{\mathrm{p}}^{2}=.83$.

We also computed mean recognition and approach response times for each spider and butterfly, and then tested the predicted effects on the level of stimuli with a mixed ANOVA with response as within and stimulus category as between factor. Congruent to the participant-based analysis, the stimulus-based analysis yielded a significant interaction between response and stimulus category, $F(1,18)=5.34, p=.03, \eta_{\mathrm{p}}{ }^{2}=.22$, indicating that spiders $(M=566 \mathrm{~ms}, S D=25)$ are recognized faster than butterflies $(M=590 \mathrm{~ms}, S D=21)$, and butterflies $(M=332 \mathrm{~ms}$, $S D=12)$ are approached faster than spiders $(M=340 \mathrm{~ms}$, $S D=17$ ). Planned contrast analyses confirm this interpretation for recognition responses, $t(18)=2.24, p=.04$, $d=.84$, but not for approach movements, $t(18)=0.90$, $p=.38$. In addition to the significant interaction and similar to the participant-based analysis, the main effect of response was significant, $F(1,18)=2,450.37, p<.001$, $\eta_{\mathrm{p}}{ }^{2}=.99$, indicating faster approach responses $(M=336 \mathrm{~ms}, \quad S D=15)$ than recognition responses $(M=578 \mathrm{~ms}, S D=26)$. The main effect of the stimulus category was not significant, $F(1,18)=1.39, p=.25$.

\section{Discussion}

Previous research has shown that positive stimuli are often recognized (Bargh et al., 1992; Unkelbach et al., 2008, 2010; see also Balota, 1994) and approached (Brendl et al., 2005; Chen \& Bargh, 1999; De Houwer et al., 2001; Krieglmeyer \& Deutsch, 2010; van Dantzig et al., 2008)

2 Independent of whether we analyze the data with the log-transformed latencies or with the latencies in ms, we find the same results.

3 After participants had completed the RaBAT, we also assessed their fear of spiders by using the German version of the SAS and FSQ. However, we found neither an influence of the SAS nor of the FSQ score on the RaBAT scores, which may be due to the fact that participants in our sample had relatively low levels of fear of spiders. 
faster than negative stimuli. The present study, however, shows that individuals recognize negative stimuli faster than positive stimuli when the negative stimuli were related to threat, but that individuals approach threatening stimuli not faster than positive stimuli.

The results illustrate an adaptive interplay between approach movements and recognition times and the importance of disentangling these two responses from each other. The described pattern was found for an analysis on the basis of the participants as well as on the basis of the stimuli. In the participant-based analysis, recognition times were faster for threatening than for positive stimuli, and approach times were even slower for the threatening than for the positive stimuli. The stimuli-based analysis revealed the same effects, although it did not detect an effect on the approach movements. As the stimuli set was limited to 10 pictures for each category, one may speculate whether an increase in the number of tested pictures would have revealed a similar effect as on the participants-based analysis. Nevertheless, it is important to note that both analyses clearly show the speed advantage of threatening stimuli in the recognition task, and they also show that recognition and approach times are not affected in the same direction by the stimulus category.

These findings are in line with a motivational account underlying approach-avoidance predispositions (Krieglmeyer \& Deutsch, 2010), proposing that the evaluation of objects triggers functional responses to change the distance between the self and that object in order to fulfill fundamental needs of survival and nurturance. Freezing and a quick avoidance movement may be the most natural responses to threatening stimuli (e.g., Rinck, Kwakkenbos, Dotsch, Wigboldus, \& Becker, 2010). However, in situations where approach is necessary a cautious and slow execution of the movement is also functional. For example, in case you identify a bird spider in your vacation rental a careful and consciously controlled approach movement might be the most functional behavior to catch the spider. The mechanism that underlies such an effect can be at least twofold. First, it might be that a slowed down approach movement is a prepared natural response to threatening stimuli. Alternatively, it may be that the approaching of threatening stimuli is slowed down because the fast approach movement of threatening stimuli, which was actually required from the participants by our instruction, does not feel right. That is the recognition of threat may have activated the probably more natural behavioral repertoire of avoiding the stimulus and thus may have inhibited the approach movement. Future research may explore whether one or even both of these explanations account for the effect.

A third alternative explanation for the slower approach movements toward threatening stimuli might be due to a previous freezing reaction. However, this explanation seems to be less likely in view of the general findings in the literature (Krieglmeyer \& Deutsch, 2010; Rinck \& Becker, 2007). These findings clearly demonstrate that avoidance movements to spiders compared to butterflies are sped up. If indeed a freezing reaction has slowed down the approach movements in our present study, one would expect that every movement that may follow should slow down as well. Since past research (Krieglmeyer \& Deutsch, 2010; Rinck \& Becker, 2007) has not found support for this assumption, we regard it as rather unlikely that freezing reactions in response to threatening stimuli have slowed down the approach movements in our present study.

An interesting question is whether the observed effects on the recognition times are driven by feelings of threat, feelings of disgust, or both. Past research has shown that beside feelings of threat, spiders, and snakes often elicit disgust also and that disgust contributes to a fast recognition of these targets similarly as threat does (Wiens et al., 2008). Our data does not allow for testing whether perceived disgust, threat, or both contribute to our findings. However, disgust and threat can be regarded as general warning feelings (e.g., Woody \& Teachman, 2000). We suppose that, on a higher level such warning feelings lead by default to functional responses such as a fast recognition (e.g., Lipp \& Waters, 2007; Tipples et al., 2002) and a fast avoidance movement (Krieglmeyer \& Deutsch, 2010; Rinck \& Becker, 2007).

A further finding of the present research was that participants took longer to recognize than to approach the presented pictures. We are careful to interpret this result as meaningful. We rather suggest that this pattern is merely dependent on the distance between the keys. With a larger keyboard (or another key closer to the screen) the difference would increase, with a smaller keyboard (or a key closer to the spacebar) it would decrease.

As far as we know, the RaBAT is the first task that used pictures, instead of words, to test differences in recognition of positive compared to negative (Genschow et al., 2013) and of positive compared to threatening stimuli (present study). Although Genschow et al. (2013) found faster recognition times of positive than of negative stimuli and we found faster recognition times of threatening than of positive stimuli in the present study, the interpretation of the findings is still limited. As pictures, but not words, were used, it may be that not the perceived valence or threat of the stimuli, but specific features of the pictures account for the found effects.

In sum, the present findings illustrate the usefulness and versatility of the RaBAT. While previous research has mainly focused on approach-avoidance and recognition responses toward threatening stimuli by measuring these components separately, we were interested in the interplay between approach movements and recognition times. Whereas previous research with the RaBAT showed parallel effects of valence on recognition and approach movement times (Genschow et al., 2013), the present findings of theoretically predicted opposite effects on the two measures clearly attest to the validity of the RaBAT and highlight the usefulness of separately measuring recognition and approach. Disentangling these components reduces noise and improves the validity of the assessment. 


\section{References}

Balota, D. A. (1994). Visual word recognition: The journey from features to meaning. In M. A. Gernsbacher (Ed.), Handbook of psycholinguistics (pp. 303-348). San Diego, CA: Academic Press.

Bamford, S., \& Ward, R. (2008). Predispositions to approach and avoid are contextually sensitive and goal dependent. Emotion, 8, 174-183.

Bargh, J. A., Chaiken, S., Govender, R., \& Pratto, F. (1992). The generality of the automatic attitude activation effect. Journal of Personality and Social Psychology, 62, 893-912.

Blanchette, I. (2006). Snakes, spiders, guns, and syringes: How specific are evolutionary constraints on the detection of threatening stimuli? The Quarterly Journal of Experimental Psychology, 59, 1484-1504.

Brendl, C. M., Markman, A. B., \& Messner, C. (2005). Indirectly measuring evaluations of several attitude objects in relation to a neutral reference point. Journal of Experimental Social Psychology, 41, 346-368.

Brosch, T., \& Sharma, D. (2005). The role of fear-relevant stimuli in visual search: A comparison of phylogenetic and ontogenetic stimuli. Emotion, 5, 360-364.

Chen, M., \& Bargh, J. A. (1999). Consequences of automatic evaluation: Immediate behavioral predispositions to approach or avoid the stimulus. Personality and Social Psychology Bulletin, 25, 215-224.

De Houwer, J., Crombez, G., Baeyens, F., \& Hermans, D. (2001). On the generality of the affective Simon effect. Cognition and Emotion, 15, 189-206.

Eder, A. B., \& Rothermund, K. (2008). When do motor behaviors (mis)match affective stimuli? An evaluative coding view of approach and avoidance reactions. Journal of Experimental Psychology: General, 137, 262-281.

Fiedler, K., Messner, C., \& Bluemke, M. (2006). Unresolved problems with the "I," the "A," and the "T": A logical and psychometric critique of the Implicit Association Test (IAT). European Review of Social Psychology, 17, 74-147.

Fredrikson, M., Annas, P., Fischer, H. Ö., \& Wik, G. (1996). Gender and age differences in the prevalence of specific fears and phobias. Behaviour Research and Therapy, 34, 33 39.

Genschow, O., Florack, A., Chib, V. S., Shimojo, S., Scarabis, M., \& Wänke, M. (2013). Reaching for the (product) stars: Measuring recognition and approach speed to get insights into consumer choice. Basic and Applied Social Psychology, $35,298-315$.

Krieglmeyer, R., \& Deutsch, R. (2010). Comparing measures of approach-avoidance behaviour: The manikin task vs. two versions of the joystick task. Cognition and Emotion, 24 , 810-828.

Lavender, T., \& Hommel, B. (2007). Affect and action: Towards an event-coding account. Cognition and Emotion, 21, 12701296.

Lipp, O. V., \& Waters, A. M. (2007). When danger lurks in the background: Attentional capture by animal fear-relevant distractors is specific and selectively enhanced by animal fear. Emotion, 7, 192-200.

LoBue, V., \& DeLoache, J. S. (2008). Detecting the snake in the grass attention to fear-relevant stimuli by adults and young children. Psychological Science, 19, 284-289.

LoBue, V., \& DeLoache, J. S. (2010). Superior detection of threat-relevant stimuli in infancy. Developmental Science, 13, 221-228.

Mineka, S., \& Öhman, A. (2002). Phobias and preparedness: The selective, automatic, and encapsulated nature of fear. Biological Psychiatry, 52, 927-937.
Morange, F., \& Bloch, H. (1996). Lateralization of the approach movement and the prehension movement in infants from 4 to 7 months. Early Development and Parenting, 5, 81-92.

Niedenthal, P. M., Barsalou, L. W., Winkielman, P., KrauthGruber, S., \& Ric, F. (2005). Embodiment in attitudes, social perception, and emotion. Personality and Social Psychology Review, 9, 184-211.

Nosek, B. A., \& Banaji, M. R. (2001). The go/no-go association task. Social Cognition, 19, 625-666.

Öhman, A., Flykt, A., \& Esteves, F. (2001). Emotion drives attention: Detecting the snake in the grass. Journal of Experimental Psychology: General, 130, 466-478.

Öhman, A., \& Mineka, S. (2001). Fears, phobias, and preparedness: Toward an evolved module of fear and fear learning. Psychological Review, 108, 483-522.

Öhman, A., \& Mineka, S. (2003). The malicious serpent snakes as a prototypical stimulus for an evolved module of fear. Current Directions in Psychological Science, 12, 5-9.

Rinck, M., \& Becker, E. S. (2007). Approach and avoidance in fear of spiders. Journal of Behavior Therapy and Experimental Psychiatry, 38, 105-120.

Rinck, M., Kwakkenbos, L., Dotsch, R., Wigboldus, D. H. J., \& Becker, E. S. (2010). Attentional and behavioural responses of spider fearfuls to virtual spiders. Cognition and Emotion, $24,1199-1206$

Rothermund, K., \& Wentura, D. (2004). Underlying processes in the implicit association test: Dissociating salience from associations. Journal of Experimental Psychology: General, $133,139-165$.

Rugg, M. D., \& Curran, T. (2007). Event-related potentials and recognition memory. Trends in Cognitive Sciences, 11, 251-257.

Seibt, B., Neumann, R., Nussinson, R., \& Strack, F. (2008). Movement direction or change in distance? Self-and objectrelated approach-avoidance motions. Journal of Experimental Social Psychology, 44, 713-720.

Strack, F., \& Deutsch, R. (2004). Reflective and impulsive determinants of social behavior. Personality and Social Psychology Review, 8, 220-247.

Tipples, J., Young, A. W., Quinlan, P., Broks, P., \& Ellis, A. W. (2002). Searching for threat. The Quarterly Journal of Experimental Psychology: Section A, 55, 1007-1026.

Unkelbach, C., Fiedler, K., Bayer, M., Stegmüller, M., \& Danner, D. (2008). Why positive information is processed faster: The density hypothesis. Journal of Personality and Social Psychology, 95, 36-49.

Unkelbach, C., von Hippel, W., Forgas, J. P., Robinson, M. D., Shakarchi, R. J., \& Hawkins, C. (2010). Good things come easy: Subjective exposure frequency and the faster processing of positive information. Social Cognition, 28, 538-555.

van Dantzig, S., Pecher, D., \& Zwaan, R. A. (2008). Approach and avoidance as action effects. The Quarterly Journal of Experimental Psychology, 61, 1298-1306.

von Hofsten, C., \& Rönnqvist, L. (1988). Preparation for grasping an object: A developmental study. Journal of Experimental Psychology: Human Perception and Performance, 14, 610-621.

Wiens, S., Peira, N., Golkar, A., \& Öhman, A. (2008). Recognizing masked threat: Fear betrays, but disgust you can trust. Emotion, 8, 810-819.

Williams, J. M. G., Watts, F. N., MacLeod, C., \& Mathews, A. (1988). Cognitive psychology and emotional disorders. Chichester, UK: Wiley.

Woody, S. R., \& Teachman, B. A. (2000). Intersection of disgust and fear: Normative and pathological views. Clinical Psychology: Science and Practice, 7, 291-311.

Zajonc, R. B. (1984). On the primacy of affect. American Psychologist, 39, 117-123. 
Received October 23, 2012

Revision received August 26, 2013

Accepted September 11, 2013

Published online November 15, 2013

\section{Oliver Genschow}

Department for Consumer and Economic Psychology Faculty for Social Sciences

University of Mannheim

Parkring 47

68159 Mannheim

Germany

E-mail ogenscho@mail.uni-mannheim.de 